\title{
The Beam Energy Scan at STAR at the Relativistic Heavy Ion Collider
}

\author{
Declan Keane for the STAR Collaboration* \\ Kent State University \\ E-mail: keane@kent.edu
}

\begin{abstract}
Phase-I of the RHIC Beam Energy Scan (BES) by the STAR experiment is based on Au $+\mathrm{Au}$ data taken in 2010 and 2011 at $\sqrt{s_{N N}}=7.7,11.5,19.6,27$ and $39 \mathrm{GeV}$, and when interpreted in conjunction with the large datasets available at 62.4 and $200 \mathrm{GeV}$, permits an initial exploration of the phase diagram of QCD matter. The three goals of BES Phase-I are as follows. 1) A search for turn-off of the promising signatures of Quark-Gluon Plasma (QGP) already reported at the top RHIC energies. 2) A search for evidence of a possible first-order phase transition such as a signature of softening of the QCD Equation of State (EOS). 3) A search for a critical end point as expected in a scenario where there is a crossover transition from hadronic matter to QGP at the highest RHIC energies, but a first-order phase transition at lower energies with finite netbaryon density. All of the BES Phase-I measurements are characterized by steeply increasing statistical error bars as the beam energy decreases, due to the characteristics of the attainable collider luminosity in BES Phase-I.
\end{abstract}

Xth Quark Confinement and the Hadron Spectrum

8-12 October 2012

TUM Campus Garching, Munich, Germany

\footnotetext{
* Speaker.
} 


\section{Introduction}

During the first decade of operation of the Relativistic Heavy-Ion Collider at Brookhaven, the focus of research was on collisions at $\sqrt{s_{N N}}=200 \mathrm{GeV}$, with a minor excursion to the moderately lower energy of $62 \mathrm{GeV}$ [1]. Data from that initial period revealed evidence for production of a deconfined partonic phase in $\mathrm{Au}+\mathrm{Au}$ collisions at top RHIC energies. However, questions remain about how nuclear matter undergoes the transition from its initial state to a deconfined QGP and then back to a hot hadronic gas. Our current level of understanding is illustrated by the conceptual phase diagram on the left in Fig. 1. The transition is believed to be a smooth crossover [2] at top RHIC energies. By colliding at progressively lower energies, it should be possible to traverse regions of increasingly high baryon chemical potential $\mu_{B}$, offering the possibility to explore the first-order phase transition and the vicinity of the associated critical point.

In 2010 and 2011, the original RHIC measurements at $\sqrt{s_{N N}}=200$ and $62.4 \mathrm{GeV}$ were supplemented by new data at $39,27,19.6,11.5$, and $7.7 \mathrm{GeV}$, forming a set of measurements normally referred to as Phase-I of the RHIC Beam Energy Scan (BES) [3]. This $\sqrt{s_{N N}}$ interval is predicted to be associated with baryon chemical potentials (at the position of the transition back to hadronic matter) in the range 25 to $\sim 450 \mathrm{MeV}$ [4]. Furthermore, the NA49 collaboration has reported evidence that the onset of deconfinement occurs close to $\sqrt{s_{N N}}=7.7 \mathrm{GeV}$ [5].

The first BES goal is to search for the turn-off of the various signals which support the interpretation that QGP has been observed at top RHIC energies. A disappearance of these signals does not automatically tell us the location of the onset of deconfinement, since reasons other than the onset of deconfinement might attenuate or obscure any individual signal as the beam energy drops, but a simultaneous disappearance of a few independent signatures would be compelling. The second BES goal is to find evidence of the system crossing a first-order phase transition. Theoretical arguments favor observables like anisotropic flow for pursuit of this goal, because such signals are a measure of the pressure in a hydrodynamic picture. The pressure is expected to go through a minimum at a bombarding energy where the system first reaches a region where there is a first-order phase transition and a relatively long-lived mixed phase [6,7]. The third BES goal is to search for a critical point, which would be accompanied by a strong increase in the susceptibilities associated with critical fluctuations. The three goals above are not ordered by importance or priority.

The STAR detector is very well suited to the challenges presented by the three goals above. Firstly, being a collider experiment, there is the basic advantage of a minimal change in the detector's acceptance as the beam energy is scanned over the range of interest. Moreover, the very large and uniform acceptance in transverse momentum, rapidity and azimuth, and especially the enhanced particle identification capabilities of the full Time-of-Flight barrel [8] added in 2010, contribute to the flexibility needed to accurately measure many different observables and the correlations between them. These characteristics are especially well suited for measurements near the bottom of RHIC's range of operating energies, where luminosity drops very steeply and many analyses are statistics-limited. This situation would be greatly improved in a future BES Phase-II, as a result of upgrades in the collider and in the STAR detector.

There have been several prior proceedings that also cover recent preliminary STAR results from BES Phase-I [9]. In order to complement these existing write-ups as much as possible, the present document has a focus on a selected subset of all the reported preliminary results, where the 
findings may turn out to have the broadest interest; this subset is discussed here in somewhat more detail than in those prior overviews, where the scope of topics was broader.
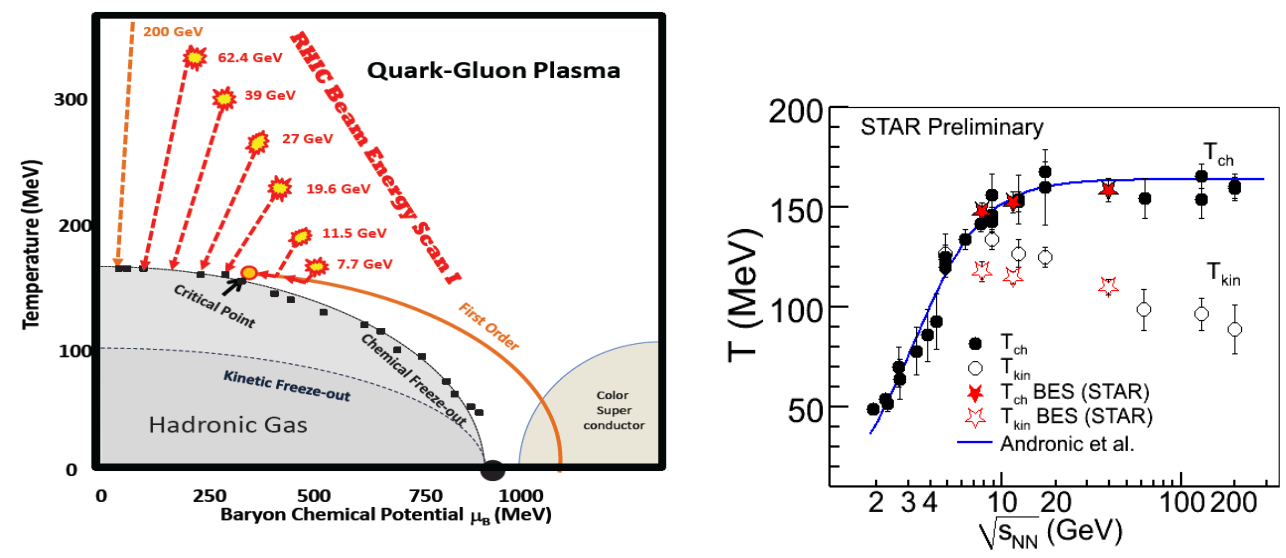

Figure 1: (Left) A conceptual QCD phase diagram, showing the region of interest for BES, in the space of $T$ vs. $\mu_{B}$. Many quantitative details, such as trajectories for specific beam energies and the position of the critical point, are based on plausible guesses. (Right): Energy dependence of temperatures for chemical freeze-out (solid symbols) and kinetic freeze-out (open symbols). The curve shows the trend from Ref. [11].

\section{Freeze-out Parameters}

Statistical-thermal models have proven successful in describing particle multiplicities in relativistic collisions of ions and particles. These models permit the use of measured particle yields as input and can generate corresponding thermodynamic parameters such as chemical freeze-out temperature $T_{\mathrm{ch}}$ and baryon chemical potential $\mu_{B}$. In general, freeze-out parameters are obtained from fits to the experimental measured ratios of produced particles, based on the statistical model THERMUS [10].

The values of the kinetic freeze-out temperature $T_{\text {kin }}$ and average flow velocity $\langle\beta\rangle$ are obtained by simultaneous fitting of pion, kaon, and proton spectral shapes to the Blast Wave model [12]. The fit parameters are $T_{\text {kin }},\langle\beta\rangle$, and flow velocity profile. The right-hand panel of Fig. 1 shows both chemical and kinetic freeze-out temperatures as a function of beam energy, and includes inferred temperatures from several experiments. It is seen that the RHIC BES measurements probe the important region where the temperature trend with energy changes from steeply rising to flat, and we also observe the onset of the chemical freeze-out temperature occurring significantly above the kinetic freeze-out.

A centrality dependence of $T_{\mathrm{ch}}$ with respect to $\mu_{B}$ is observed for the first time at the lower energies. Work is ongoing for the two more recent datasets at 19.6 and $27 \mathrm{GeV}$. The $\mu_{B}$ values at 7.7, 11.5 and $39 \mathrm{GeV}$ are within the $400-100 \mathrm{MeV}$ range. The critical region in $\mu_{B}$ has been predicted to span on the order of $100 \mathrm{MeV}$, which suggests that the overall program of BES measurements to date will offer reasonable coverage below $\mu_{B} \sim 400 \mathrm{MeV}$ when the two energy points measured in the year 2011 (19.6 and $27 \mathrm{GeV})$ are included in the analysis. On the other hand, the five energies that constitute the existing BES dataset were shifted somewhat relative to 
the original planned energy points, due to the priority of avoiding energy ranges where RHIC was not able to simultaneously provide collisions at both STAR and PHENIX. On the scale of $\mu_{B}$, there is a larger gap than normal between the values corresponding to the beam energies 11.5 and 19.6 $\mathrm{GeV}$, and there have been some theory-based arguments that an additional intermediate energy point is needed because otherwise, the QCD critical point could be missed. Fortunately, a recent insight by RHIC accelerator experts has uncovered a new mode of RF operation that now permits collisions at both experiments when running at $\sqrt{s_{N N}}=14.6 \mathrm{GeV}$. A new run at this beam energy is planned to fill-in the anomalous gap in $\mu_{B}$.

\section{Net-Proton Directed Flow}
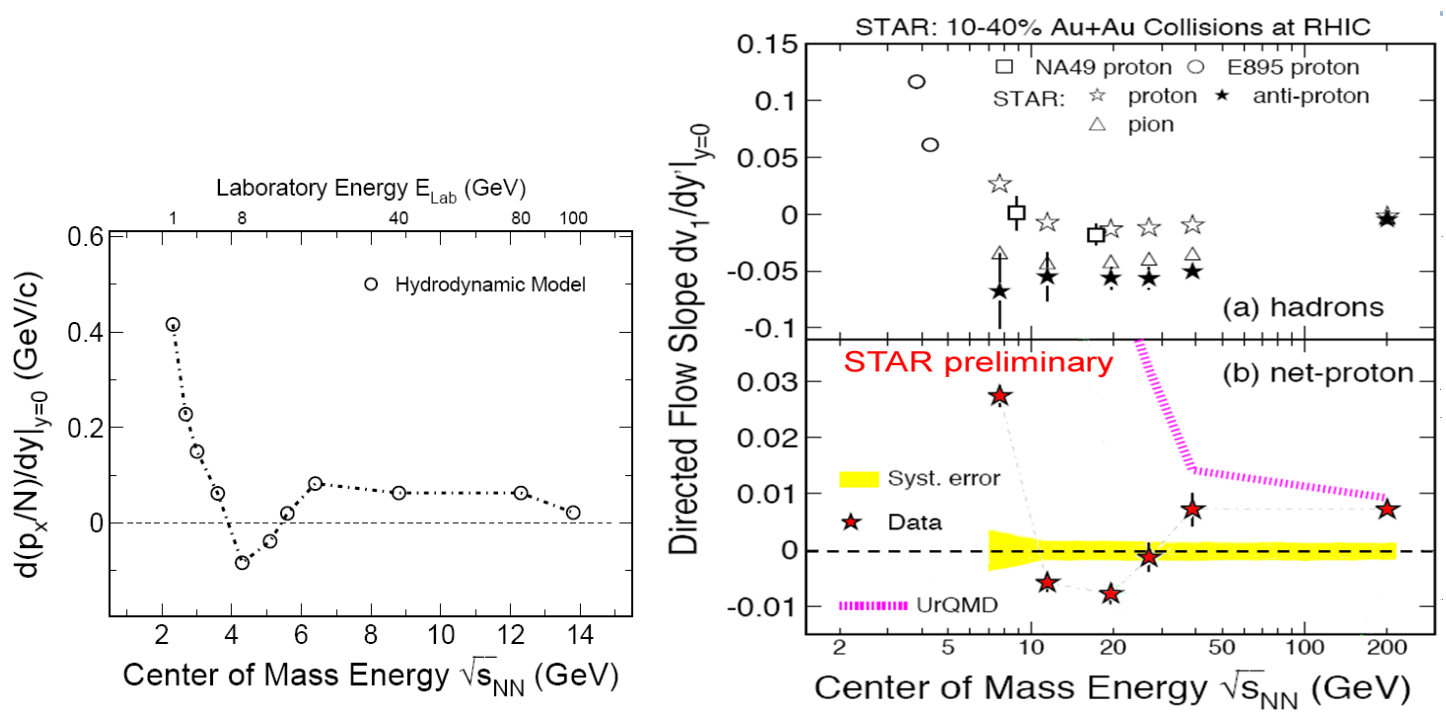

Figure 2: Left: Directed flow prediction as a function of beam energy, based on a three-fluid hydrodynamic model [7] whose EOS incorporates a first-order phase transition. Right: Panel (a) shows the slope of directed flow vs. rapidity for protons, antiprotons, and pions as a function of beam energy. Panel (b) on the right presents the proton signal with the antiproton signal subtracted after appropriate scaling. The prediction of the UrQMD transport model is also plotted. The STAR data points show only statistical errors and the shaded band is an estimate of systematic uncertainties.

Directed flow excitation functions have been proposed by the Frankfurt group as promising observables for uncovering evidence of crossing a first-order phase transition, based on hydrodynamic calculations [6, 14, 7]. Figure 2 (left panel), based on a 3-fluid hydrodynamic model [7] presents an observable closely related to the directed flow quantity $v_{1}$, for protons as a function of beam energy. A first-order phase transition leads to a softening of the EOS, and this in turn causes proton directed flow to change sign from positive to negative near $\sqrt{s_{N N}}=4 \mathrm{GeV}$. The directed flow prediction crosses back to positive again as the beam energy increases further. This phenomenon is sometimes referred to as a "collapse of proton flow" and the minimum in directed flow is said to correspond to the "softest point" in the EOS, where a relatively long-lived mixed phase occurs. It is desirable to use identified protons for such measurements, because in models 
with just conventional hadronic physics, produced particles like pions can have a directed flow that is opposite in direction from protons and this complicates the interpretation of data if all charged particles are included [15].

The directed flow excitation function measured by STAR for protons, antiprotons and pions near mid-rapidity is presented in Fig. 2 (right). The plotted quantity is $d v_{1} / d y^{\prime}$, where the primed quantity $y^{\prime}$ refers to normalized rapidity $y / y_{\text {beam. }}$. The slope is the linear term $F$ in a cubic fit, where $v_{1}=F y^{\prime}+F_{3} y^{\prime 3}$. The energy dependence of proton $d v_{1} / d y^{\prime}$ involves an interplay between the directed flow of protons associated with baryon number transported from the initial state to the vicinity of mid-rapidity, and the directed flow of protons from particle-antiparticle pairs produced near mid-rapidity. Obviously, the second mechanism increases strongly with beam energy, and it is helpful in interpretation to distinguish between the two as far as possible. We define $F_{\text {net }}$ based on an equation in which the measured slope for all protons is written $F=r F_{\bar{p}}+(1-r) F_{\text {net } p}$, where $r$ is the observed ratio of antiprotons to protons among the analyzed tracks at each beam energy. An interpretation of this quantity is suggested by our observation that $v_{1}(y)$ is almost the same for $\pi^{+}$and $\pi^{-}$and for $K^{+}$and $K^{-}$. In fact, they are indistinguishable within errors at higher energies, and are only slightly different at 11.5 and $7.7 \mathrm{GeV}$. Thus the suggested interpretation is that $F_{\bar{p}}$ is a proxy for directed flow from produced protons, and this guides our inference that the net-proton slope $F_{\text {net } p}$ isolates as far as possible the contribution of the initial-state baryonic matter.

The lower panel on the right in Fig. 2 reveals that the inferred $v_{1}$ slope for net protons becomes negative with good statistical significance at 11.5 and $19.6 \mathrm{GeV}$, while it is consistent with zero at $27 \mathrm{GeV}$ and positive at $7.7 \mathrm{GeV}$ and above $27 \mathrm{GeV}$, including at $200 \mathrm{GeV}$. In contrast, the UrQMD transport model shows a positive slope at all energies for this observable. Thus there is no hint of this remarkable non-monotonic behavior in a hadronic model that has a good record of reproducing observed trends at least at a qualitative level. AMPT model predictions have also been generated, but we are still investigating possible anomalies in the model output and therefore we do not yet plot these predictions in Fig. 2.

The beam energy region where we observe the double sign change coincides with a high degree of stopping, and lies just above the region where the spectator matter separates from the participants quickly enough so that it no longer influences flow in the midrapidity zone [13]. Nuclear transport models ought to clarify whether or not purely hadronic physics could account for the observed double sign change, and it would be reassuring to have predictions from more than one model. Thus, a definitive physics conclusion informed by one transport model comparison may be premature. To better understand the possible role and relevance of stopping, measurements of net-proton $v_{1}$ slope as a function of centrality are needed, but current statistics are inadequate for this purpose. This strongly motivates the proposed higher-statistics measurements of BES Phase-II.

Overall, we conclude that the prominent dip and its associated double sign change bears a striking resemblance to predicted signatures of a softening of the EOS associated with a first-order phase transition, and indeed is more prominent than some such predictions $[6,7,14,16]$. The observed beam energy of the minimum is about a factor 4 higher than the hydro prediction shown on the left in Fig. 2. At the lower energy of the minimum in the hydro calculation, the difference between net protons and all protons is negligible. Possible explanations unrelated to the EOS, as discussed above, may remain as viable alternatives until further experimental and theoretical investigations are carried out. 


\section{The Chiral Magnetic Effect}

There are still many open questions related to the non-trivial structure of the QCD vacuum. The generation of mass from spontaneous chiral symmetry breaking, and topological solutions are relevant to this discussion [17]. The observation of a correlation that can be interpreted as a signal of event-by-event local parity violation (LPV) in strong interactions [18] lends support to current theoretical understanding, and has an immediate impact, not just on relativistic heavy ion physics, but on all spheres of physics touched by QCD (high energy physics, astrophysics, cosmology, etc.)

The signal of interest assumes the following chain of circumstances. In non-central heavy-ion collisions, a large orbital angular momentum vector $(L)$ exists at $90^{\circ}$ to the reaction plane, leading to an exceptionally intense localized magnetic field $\left(\sim 10^{15} \mathrm{~T}\right)$. If the system is deconfined, there can be strong parity-violating domains, and different numbers of quarks of left- and right-handed helicity, leading to preferential emission of like-sign charged particles along $L$. The phenomenon is sometimes called the Chiral Magnetic Effect (CME) [19], and has been studied in lattice QCD [20].

In the azimuthally anisotropic emission of particles, $\left(d N_{ \pm} / d \phi\right) \propto 1+2 a_{ \pm} \sin \left(\phi-\Psi_{\mathrm{RP}}\right)+$ .... the coefficient $a$ represents the size of the parity-violating signal, and the remaining terms (not shown explicitly) are the familiar ones with coefficients $v_{n}$ for directed and elliptic flow, etc. However, the coefficient $a$ averages to zero when integrated over many parity-violating domains in many events. In the presence of parity violation, a non-zero average signal can be obtained by forming a correlation between pairs of emitted particles relative to the reaction plane, in the form $\gamma=\left\langle\cos \left(\phi_{\alpha}+\phi_{\beta}-2 \psi_{\mathrm{RP}}\right)\right\rangle$ where $\alpha$ and $\beta$ denote the particle type: $\alpha, \beta=+,-$. The observed results [18] are consistent with the expected signal for local parity violation, especially the centrality dependence. There are caveats attached to this observation - the expected parity violation is parity-odd, whereas the only accessible observable to measure it, $\gamma$, is parity-even. This means that effects not related to parity violation (e.g., jets and resonances) can contribute to the measured signal. It has been argued that there is no known background, or effect predicted by existing eventgenerating models, that could account for the observed signals [18]. This interpretation has been challenged by an argument that a combination of elliptic flow and charge conservation effects can reproduce the observed signal [21]. The counter argument is that the critique in Ref. [21] is too simplistic, and this line of investigation is still continuing.

The energy scan has the potential to open up new physics related to chiral magnetism, since CME is generally accepted as needing deconfinement to happen [19]. So CME is a possible deconfinement signal that we expect to turn-off at some point if we scan down low enough in energy. Keeping in mind that the duration of the intense magnetic field becomes longer as the beam energy is lowered, it is plausible that the CME signal will increase steadily as we scan down, and then reverse its trend more abruptly upon passing a threshold energy for deconfinement [22]. On the other hand, the known background effects are expected to have a quite different energy dependence. There remains a pressing need for theoretical calculations of the energy dependence of chiral magnetism, so that quantitative predictions can be generated. Regardless of the various uncertainties in theory and in experimental interpretation as discussed above, it is of considerable interest to measure the beam energy dependence of the correlations first reported in Ref. [18].

Figure 3 presents the extension of the analysis of Ref. [18] to the BES energies. For compar- 


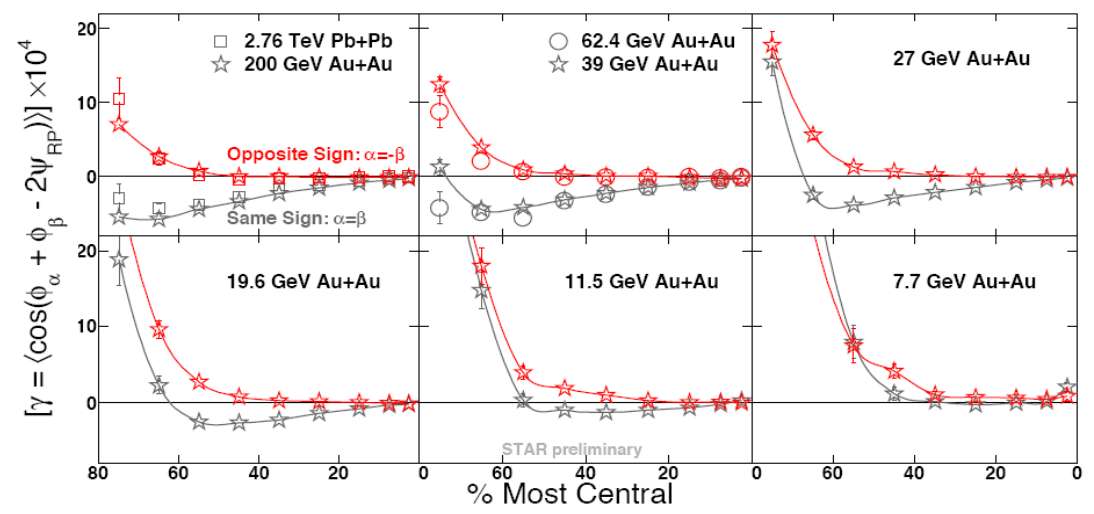

Figure 3: The correlator $\gamma$ as a function of centrality for Au+Au collisions from $200 \mathrm{GeV}$ to $7.7 \mathrm{GeV}$. For comparison, we also show ALICE results for $\mathrm{Pb}+\mathrm{Pb}$ at $2.76 \mathrm{TeV}$ [23]. Plotted errors are statistical only.

ison, we also show the ALICE results for $\mathrm{Pb}+\mathrm{Pb}$ collisions at $2.76 \mathrm{TeV}$ [23]. As can be seen in Fig. 3, the $\gamma$ signals are very similar in collisions throughout the beam energy range $\sqrt{s_{N N}}=2.76$ $\mathrm{TeV}$ down to to $19.6 \mathrm{GeV}$. However, changes in the correlation function are seen at lower energies: $\sqrt{s_{N N}}=11.5$ and $7.7 \mathrm{GeV}$, where the difference between same-sign and opposite-sign $\gamma$ fades away at all collision centralities. As discussed above, one possible explanation for this fading away would be the disappearance of the CME due to a turn-off of deconfinement at lower BES energy points.

In order to further test the interpretations, STAR has carried out a measurement with $U+U$ collisions, where the large deformation of uranium nuclei leads to specific consequences for the elliptic flow and its fluctuations in very central collisions, which in turn presents new opportunities to make a cleaner distinction between a true CME signal and the possible background signals associated with elliptic flow. A progress report on a $U+U$ analysis along these lines has been presented at Quark Matter 2012 [24].

\section{Summary and Outlook}

Based on preliminary analyses of BES Phase-I, we report the status of STAR's search for possible signals in the three main categories set out in the introduction. Prior proceedings cover a large fraction of the available BES measurements [9], whereas the present document has a narrower focus on a promising subset - data for locations of freeze-out in the space of temperature and baryon chemical potential, measurements of the net-proton directed flow which shows a striking non-monotonic behavior with energy, and the disappearance at low energies of the difference between same-sign and opposite-sign correlations that are argued to arise from the Chiral Magnetic Effect. Findings to date are not conclusive, but strongly motivate further measurements, especially at lower BES energies where statistical errors are large. The proposed BES Phase-II will overcome these limitations by improved collider luminosity and STAR detector upgrades. 


\section{References}

[1] J. Adams et al. (STAR Collaboration), Nucl. Phys. A 757, 102 (2005).

[2] F. Karsch et al., Nucl. Phys. Proc. Suppl. 129, 614 (2004); M. Cheng et al., Phys. Rev. D 79, 074505 (2009) and references therein.

[3] B. I. Abelev et al. (STAR Collaboration), arXiv:1007.2613.

[4] J. Cleymans, H. Oeschler, K. Redlich and S. Wheaton, Phys. Rev. C 73, 034905 (2006).

[5] C. Alt et al. (NA49 collaboration), Phys. Rev. C 77, 024903 (2008).

[6] D. H. Rischke et al., Heavy Ion Phys. 1, 309 (1995).

[7] H. Stöcker, Nucl. Phys. A750, 121 (2005).

[8] W. Llope for the STAR Collaboration, Nucl. Instr. Meth. A 661, S110 (2012).

[9] L. Kumar for the STAR collaboration, Proc. Quark Matter 2012, Washington DC (Nucl. Phys. A, in press), preprint arXiv:1211.1350; D. Cebra for the STAR collaboration, ibid.; Y. Pandit for the STAR collaboration, ibid., preprint arXiv:1210.5315; D. McDonald for the STAR collaboration, ibid. preprint arXiv:1210.7023; S. Das for the STAR collaboration, ibid., preprint arXiv:1210.6099; A. Schmah for the STAR collaboration, Proc. 2012 FAIRNESS workshop, Hersonissos, Greece (J. Phys.: Conf. Series, in press).

[10] S. Wheaton, J. Cleymans, and M. Hauer, Comp. Phys. Communications 180, 84 (2009).

[11] A. Andronic, P. Braun-Munzinger and J. Stachel, Nucl. Phys. A 834, 237 (2010).

[12] E. Schnedermann et al., Phys. Rev. C48, 2462 (1993).

[13] P. Chung et al., Phys. Rev. C 66, 021901 (2002).

[14] J. Brachmann et al., Phys. Rev. C 61, 024909 (2000).

[15] R. J. Snellings, H. Sorge, S. A. Voloshin, F. Q. Wang and N. Xu, Phys. Rev. Lett 84, 2803 (2000).

[16] N. Herrmann, J. P. Wessels, and T. Wienold, Annu. Rev. Nucl. Part. Sci. 49, 581 (1999); L. P. Csernai and D. Rohrich, Phys. Lett. B 458, 454 (1999); P. Bozek and I. Wyskiel, Phys. Rev. C 81, 054902 (2010).

[17] E. V. Shuryak and T. Schafer, Ann. Rev. Nucl. Part. Sci. 47, 359 (1997); D. Diakonov, Prog. Part. Nucl. Phys. 51, 173 (2003).

[18] B. I. Abelev et al. (STAR collaboration), Phys. Rev. Lett. 103, 251601 (2009); Phys. Rev. C 81, 054908 (2010).

[19] D. Kharzeev, Phys. Lett. B 633, 260 (2006); D. E. Kharzeev, L. D. McLerran and H. J. Warringa, Nucl. Phys. A 803, 227 (2008).

[20] P. V. Buividovich, M. N. Chernodub, E. V. Luschevskaya and M. I. Polikarpov, Phys. Rev. D 80, 054503 (2009); M. Abramczyk, T. Blum, G. Petropoulos and R. Zhou, arXiv:0911.1348; P. V. Buividovich, E. V. Lushchevskaya, M. I. Polikarpov and M. N. Chernodub, JETP Lett. 90, 412 (2009).

[21] S. Pratt, Phys. Rev. C 83, 014913 (2011); S. Pratt, S. Schlichting, S. Gavin, Phys. Rev. C 84, 024909 (2011).

[22] S. A. Voloshin, arXiv:1003.1127.

[23] B. Abelev et al. (ALICE Collaboration), arXiv:1207.0900.

[24] G. Wang for the STAR Collaboration, Proc. Quark Matter 2012, Washington DC (Nucl. Phys. A, in press). 